characteristics interact so that combinations of factors carry cumulative risk for CVD? (iii) are associations explained by early life predictors of CVD risk.

Design: Birth cohort.

Setting: England, Scotland and Wales.

Participants: 7916 men and women in the 1958 British birth cohort who were in paid employment at $45 \mathrm{y}$.

Main Outcome Measures: Body mass index (BMI), waist circumference (WC), blood pressure (BP), triglycerides, total and high density lipoprotein (HDL) cholesterol, glycosylated haemoglobin $(\mathrm{HbA} 1 \mathrm{c})$, and inflammatory factors: fibrinogen, C-reactive protein (CRP)

Results: Night work was associated with adverse levels of most outcomes examined (except BP and total cholesterol). Working $\geqslant 48 \mathrm{~h} /$ week was associated with BMI and WC only; low job control with $\mathrm{HDL}, \mathrm{HbA1c}$, and inflammatory factors; low demands, rather than high demands, with systolic BP, triglycerides, HDL and inflammatory factors. Several work factor/CVD associations were weakened when mutually adjusted for each other and an interaction between night work and low demands was commonly found. Adjustment for childhood factors up to $16 \mathrm{y}$ explained a substantial proportion of the associations. To illustrate, for BMI, adjustment for a range of childhood factors reduced the associations for night work/low demands $\left(0.78 \mathrm{~kg} / \mathrm{m}^{2}, 95 \%\right.$ CI 0.35 to 1.21$)$ and working $\geqslant 48 \mathrm{~h} /$ week $\left(0.31 \mathrm{~kg} / \mathrm{m}^{2},-0.01\right.$ to 0.62$)$ by $50 \%$ and $22 \%$, respectively. However, BMI at $16 \mathrm{y}$ accounted for $45 \%$ of the association between working $\geqslant 48 \mathrm{~h} /$ week and BMI at $45 \mathrm{y}$ (adjustment for some childhood factors strengthened the association between work hours and BMI).

Conclusions: Initial findings suggest that childhood factors up to the age of $16 \mathrm{y}$ explain a large proportion of the cross-sectional associations seen for work characteristics and risk factors for CVD in mid-adulthood suggesting that associations arise in part from social and health disadvantage originating earlier in life.

\section{LIFECOURSE PREDICTORS OF ADULT FIBRINOGEN CONCENTRATIONS: THE NEWCASTLE THOUSAND FAMILIES BIRTH COHORT}

${ }^{1}$ MS Pearce, ${ }^{2} \mathrm{~A}$ Ahmed, ${ }^{1} \mathrm{PWG}$ Tennant, ${ }^{3} \mathrm{~L}$ Parker, ${ }^{1} \mathrm{NC}$ Unwin. ${ }^{1}$ Institute of Health and Society, Newcastle University, Newcastle upon Tyne, UK; ${ }^{2}$ London School of Hygiene and Tropical Medicine, London, UK; ${ }^{3}$ Departments of Medicine and Paediatrics, Dalhousie University, Halifax, NS, Canada

\section{doi:10.1136/jech.2009.096701d}

Objectives: To investigate the relative influences of early life and later determinants of plasma fibrinogen concentrations at age 4951 years.

Design: Follow-up study of the Newcastle Thousand Families birth cohort established in 1947.

Participants: 173 men and 221 women who attended a clinical examination between October 1996 and December 1998 and also gave blood samples.

Main Outcome Measure: Concentration of plasma fibrinogen. This was analysed, using linear regression, in relation to a range of variables at different stages of life, including family history of cardiovascular disease, birth weight (standardised for gestational age and sex), duration breast fed, housing conditions at birth, history of childhood illness, cigarette smoking history, alcohol consumption, percent body fat, physical activity levels, and socioeconomic status both at birth and in adulthood.

Results: Poorer housing conditions at birth $(p=0.001)$, longer duration breast fed $(p=0.025)$ and higher adult alcohol consumption $(p=0.002)$ were all significant independent predictors of lower plasma fibrinogen concentration at age 49-51 years. In contrast, increasing body fat percentage $(p<0.001)$ and being a current smoker $(p<0.001)$ were both independently predictive of a significantly higher fibrinogen concentration. No association was observed between plasma fibrinogen concentration and standardised birth weight or with time since stopping smoking the former smokers. Three significant interactions on adult fibrinogen levels were observed. (1) The effect of being a current smoker, relative to never smokers, was highest among those from the poorest quality houses at birth. (2) The effect of percent body fat was lower among never smokers. (3) The effect of percent body fat was greater among those with the highest alcohol consumption at age 49-51. A full path diagram, exploring the relative contributions, including an exploration of indirect pathways, will be presented.

Conclusions: Concentration of plasma fibrinogen in adulthood is influenced by a range of factors from different stages of life. Although birth weight was not a predictor, there were significant associations with housing conditions in early life and duration breast fed. Regardless, the total variation explained by early life factors was less than half of that explained by adult risk factors. Therefore, modification of adult exposures, particularly body fat percentage and smoking, would be the most likely way to reduce the concentration of plasma fibrinogen in adulthood, which may also reduce the risk of cardiovascular disease.

\section{Maternal health}

\section{SOCIAL AND BIOLOGICAL DETERMINANTS OF REPRODUCTIVE SUCCESS IN SWEDISH MALES AND FEMALES BORN 1915- 1929}

${ }^{1}$ A Goodman, ${ }^{2}$ Koupil. 'Department of Epidemiology and Population Health, London School of Hygiene and Tropical Medicine, London, UK; ${ }^{2}$ Centre for Health Equity Studies, Stockholm University/Karolinska Institute, Stockholm, Sweden

\section{doi:10.1136/jech.2009.096701e}

Objective: To investigate whether early life characteristics predict subsequent reproductive success in a post-demographic transition population; to ascertain the pathways mediating any observed effects; and to examine whether any of the above effects are genderspecific.

Design: Multi-generational data from a representative, populationbased birth cohort and using linkage to routinely collected data.

Setting: Uppsala, Sweden.

Participants: 13666 individuals born in Uppsala university hospital between 1915 and 1929, who were traced and linked to all registered descendants up to 2002.

Characteristics Measured at Birth: Birthweight for gestational age, preterm birth, birth multiplicity, birth order, mother's age, mother's marital status and family socio-economic position.

Measures of Reproductive Success: Primary measures: number of children; number of grandchildren. Secondary measures of the pathways to reproductive success: survival to age 15 , survival from age 15 to age 50; probability of marriage; number of children within marriage; number of grandchildren at a given number of children. Results: Reproductive success was associated with both social and biological characteristics at birth, and the effects of these characteristics were mediated via both mortality and fertility. In both sexes, a higher birthweight for gestational age, a term birth and a younger mother were independently associated with a greater number of descendants. A married mother and higher family socioeconomic position were also associated a greater number of descendants in males (but not females), while in females (but not males) higher birth order was associated with higher reproductive success. These differences between the genders were mediated by the differential effects upon the probability of marriage in men and women. Probability of marriage was also affected by a range of other characteristics at birth including a lower probability of marriage for individuals of low birthweight and males who were 
preterm. Number of grandchildren increased with increasing number of children in both sexes, providing no evidence for a trade-off between quantity of offspring and their subsequent reproductive "quality".

Conclusions: Early life characteristics can affect reproductive success even in post-demographic transition populations. These effects operate via multiple pathways and include "biological" characteristics such as birthweight having an effect via social facts such as adult marital status. These findings can inform analyses of reproductive career as a determinant of health in later life in this and other similar populations. They also generate hypotheses regarding the potential long term consequences of adverse early environments in concurrent cohorts around the world.

\section{TRENDS IN MATERNAL OBESITY AND HEALTH INEQUALITIES IN A NATIONALLY REPRESENTATIVE SAMPLE OF 619323 BIRTHS IN ENGLAND, UK, 1989-2007}

${ }^{1} \mathrm{~N}$ Heslehurst, ${ }^{2} \mathrm{~J}$ Rankin, ${ }^{3} \mathrm{~J}$ Wikinson, ${ }^{4} \mathrm{CD}$ Summerbell. ${ }^{1}$ Health and Social Care Research Institute, University of Teesside, Middlesbrough, Tees Valley, UK; ${ }^{2}$ Institute of Health and Society, Newcastle University, Newcastle upon Tyne, UK: ${ }^{3}$ North East Public Health Observatory, Durham University, Stockton, UK; ${ }^{4}$ School of Medicine and Health, Durham University, Stockton, UK

\section{doi:10.1136/jech.2009.096701f}

Objectives: Maternal obesity has serious implications to the health of both mothers and infants, including maternal and neonatal death, stillbirth, congenital anomalies, poor breastfeeding rates, and obesity in the offspring. There are also additional complications during antenatal, intrapartum, and postnatal periods which impact on maternity services. However, there is an absence of national statistics for maternal obesity in the UK. This study is the first to describe a nationally representative maternal obesity research dataset in England.

Design of the Study: Descriptive epidemiological study using routinely collected data.

Setting: 34 maternity units in England.

Participants: 619323 women who delivered at the maternity units sampled, between January 1989 and December 2007.

Main Outcome Measures: Trends in first trimester maternal BMI status over time and geographical distribution of maternal obesity by Government Office Regions in England. Demographics of the population were analysed to identify any maternal obesity associated health inequalities, including maternal age, parity, ethnic group, deprivation, and employment. All demographics were tested for multicollinearity. Logistic regression adjusted for all included demographics as confounders.

Results: The demographic characteristics of the study population were representative when compared to census and deprivation data. Obesity in the first trimester of pregnancy is significantly increasing over time, having more than doubled from $7.6 \%$ to $15.6 \%$ over the 19 years studied $(p<0.001)$. There is significant geographic variation in the incidence of maternal obesity, with the West Midlands, Yorkshire and the Humber and North East Government Office Regions having higher than national average incidence of first trimester obesity. There are health inequalities associated with maternal obesity, including increased odds of being obese with increasing age (1.02, 95\% CI 1.02 to 1.02$)$, parity $(1.17,95 \%$ CI 1.16 to 1.18$)$, black ethnic group $(1.78,95 \%$ CI 1.70 to 1.87$)$, and deprivation $(2.20,95 \%$ CI 2.13 to 2.28$)$. There is also an association between super morbid obesity and unemployment $(1.50,95 \% \mathrm{CI}$ 1.12 to 2.02 ).

Conclusions: The increase in maternal obesity at booking has yielded an additional 47500 women per year requiring high dependency care in England. The demographics of women most at risk of first trimester obesity highlight health inequalities associated with maternal obesity which need to be addressed.

\section{INHERITED RISK OF PRE-ECLAMPSIA: USING TWO APPROACHES FOR ANALYSIS}

S Bhattacharya, EA Raja, DM Campbell, AJ Lee. Division of Applied Medicine, University of Aberdeen, Aberdeen, UK

doi:10.1136/jech.2009.096701g

Background: Several previous research reports have suggested a genetic predisposition to pre-eclampsia but none have demonstrated the effect separately in nulliparous and parous women in the context of other risk or protective factors.

Objective: To assess the magnitude of genetic predisposition to pre-eclampsia with reference to other risk factors in nulliparous and parous women.

Material and Method: The Aberdeen Maternity and Neonatal Databank records all pregnancy and delivery details occurring in Aberdeen, Scotland since 1950. It has now become possible to link pregnancy records of mothers and grandmothers to those of the daughters. Using a nested case control design within this intergenerational cohort, statistical modelling was done with known risk/protective factors for pre-eclampsia, separately for nulliparous and parous women. Conditional logistic regression was used to compare characteristics between parous pre-eclamptics and year and parity matched normotensive controls. In a separate analysis, including all parous women, we used a multilevel approach based on Generalised Estimating Equation (GEE) and specified the link function as binomial. We assumed a working exchangeable correlation of having preeclampsia within a daughter in her pregnancies. Odds ratios (OR) and 95\% CI were estimated through GEE with the use of robust standard errors.

Results: There were 34970 mother-daughter pairs. Of the daughters, there were 1248 nulliparous and 448 parous preeclamptics. For nulliparous women, the risk factors remaining in the stepwise model were mother's history of pre-eclampsia (OR $2.13,95 \%$ CI 1.57 to 2.89 ), booking BMI $>30 \mathrm{~kg} / \mathrm{m}^{2}$ (OR 2.06, $95 \%$ CI 1.68 to 2.52), age, gestation period, and booking diastolic blood pressure. Smoking $\sim 10$ cigarettes a day was protective against preeclampsia (OR 0.52 , 95\% CI 0.44 to 0.62). For multiparae, the risk factors included pre-eclampsia in the initial pregnancy (OR 8.80, 95\% CI 1.54 to 50.23), advanced age at delivery (OR 3.09, 95\% CI 1.69 to 5.66 ) and BMI $>30 \mathrm{~kg} / \mathrm{m}^{2}$ (OR 2.61, 95\% CI 1.62 to 4.20 ). Smoking 10 or more cigarettes per day was protective (OR 0.57 , $95 \%$ CI 0.35 to 0.94 ). A history of maternal pre-eclampsia was not independently associated with an increased risk of development of pre-eclampsia in the multiparae after adjusting for other covariates. Results were similar using the GEE approach.

Conclusion: In nulliparous women, a history of maternal preeclampsia was associated with more than doubling of risk of preeclampsia. In multiparae, this association was not observed, although a history of pre-eclampsia in a previous pregnancy was strongly associated with increased risk, suggesting genetic susceptibility.

\section{BREASTFEEDING IS ASSOCIATED WITH IMPROVED CHILD COGNITIVE DEVELOPMENT: EVIDENCE FROM THE UK MILLENNIUM COHORT STUDY}

${ }^{1} \mathrm{MA}$ Quigley, ${ }^{1} \mathrm{C}$ Hockley, ${ }^{1} \mathrm{C}$ Carson, ${ }^{2} \mathrm{Y}$ Kelly, ${ }^{3} \mathrm{M}$ Renfrew, ${ }^{4} \mathrm{~A}$ Sacker. ${ }^{1}$ National Perinatal Epidemiology Unit, University of Oxford, Oxford, UK; ${ }^{2}$ Department of Epidemiology and Public Health, University College London, London, UK; ${ }^{3}$ Mother and Infant Research Unit, University of York, Heslington, York, UK; ${ }^{4}$ Institute for Social and Economic Research, University of Essex, Colchester, Essex, UK

doi:10.1136/jech.2009.096701h

Objective: To assess the relationship between breastfeeding and child cognitive development, and whether this relationship varies according to prematurity. 